Referencia para citar este artículo: Ríos-Flórez, J. A., Álvarez-Londoño, L. M., David-Sierra, D. E., \& Zuleta-Muñoz, A. C. (2018). Influencia del nacimiento pretérmino en procesos conductuales y emocionales de niños en etapa escolar primaria. Revista Latinoamericana de Ciencias Sociales, Niñez y Juventud, 16(1), 177-197. doi:10.11600/1692715x.16110

\title{
Influencia del nacimiento pretérmino en procesos conductuales y emocionales de niños en etapa escolar primaria*
}

\author{
JORGE ALEXANDER RÍOS-FLÓREZ ${ }^{* *}$ \\ Director del Grupo de Estudio e Investigación en Neurociencias Hippocampus, Colombia. \\ LUISA MARÍA ÁLVAREZ-LONDOÑo ${ }^{* * *}$ \\ Miembro del Grupo de Estudio e Investigación en Neurociencias Hippocampus, Colombia. \\ DIANA ESTEFANÍA DAVID-SIERRA ${ }^{* * * *}$ \\ Miembro del Grupo de Estudio e Investigación en Neurociencias Hippocampus, Colombia. \\ Adriana CECILIA ZulETA-MUÑOZ \\ Miembro del Grupo de Estudio e Investigación en Neurociencias Hippocampus, Colombia.
}

\section{Artículo recibido en agosto 12 de 2016; artículo aceptado en enero 17 de 2017 (Eds.)}

- Resumen (analítico): en esta investigación buscamos establecer las principales características conductuales que pueden manifestarse en niños y niñas entre seis a diez años de edad, con antecedente de nacimiento prematuro. Utilizamos la batería de pruebas BASC (del inglés Behavior Assessment System for Children), la lista de chequeo de los criterios de hiperactividad del DSM-5 y la prueba Caras-R. Aplicamos las pruebas a una muestra de 80 niños y niñas nacidos pretérmino (muestra clínica) y a 80 niños y niñas nacidos a término (muestra control). Analizamos la información a través del software estadístico SPSS ${ }^{T M}$ versión 22, y el software de apoyo para la evaluación neuropsicológica de la conducta humana. En relación con el TDAH, son más frecuentes los casos de inatención o impulsividad en los niños y niñas prematuros, los cuales son percibidos en sus hogares con mayores conductas atípicas, sintomas de hiperactividad, episodios de aislamiento y sintomatología característica de depresión y cuadros de somatización.

Palabras clave: prematurez, conducta, emociones, TDAH, atención (Tesauro de Ciencias Sociales de la Unesco).

\footnotetext{
* Este artículo de investigación científica y tecnológica. Gran Área: Neurociencias; Área: Neuropsicología; Subárea: Aprendizaje. Presenta resultados de la investigación "Neuropsicología del niño en condición de nacimiento prematuro y en edad escolar", financiada con recursos personales; realizada entre el 26 de enero de 2015 y el 30 de Julio del 2016, desde la Línea de investigación en Neurodesarrollo y Trastornos neurológicos en la infancia del Grupo de Estudio e Investigación en Neurociencias Hippocampus, Medellín.

** Psicólogo de la Universidad Pontificia Bolivariana, Especialista en Evaluación y Diagnóstico Neuropsicológico y Magíster en Neuropsicología Clínica de la Universidad de San Buenaventura, Bogotá. Doctorando en Psicobiología de la Universidade Federal do Rio Grande do Norte, Brasil. Profesor-Investigador, director del Grupo de Estudio e Investigación en Neurociencias Hippocampus, Colombia. Orcid: 0000-0002-8367-8225. Índice H5: 1. Correo electrónico: alexanderriosflorez@gmail.com

*** Psicóloga-Investigadora, miembro adjunto del Grupo de Estudio e Investigación en Neurociencias Hippocampus. Orcid: 0000-0001-9655-9156. Correo electrónico: luisamaria1481@hotmail.com

**** Psicóloga-Investigadora, miembro adjunto del Grupo de Estudio e Investigación en Neurociencias Hippocampus. Orcid: 0000-0003-0037-3125. Correo electrónico: diani_david18@hotmail.com

***** Psicóloga-Investigadora, miembro adjunto del Grupo de Estudio e Investigación en Neurociencias Hippocampus. Orcid: 0000-0002-4123-8037. Índice H5: Correo electrónico: adrizul85@hotmail.com
} 


\section{Influence of preterm births on behavioral and emotional processes of primary school age children}

- Abstract (analytical): This research aimed to establish the main behavioral characteristics that can be presented by children aged between 6-10 years old who were born prematurely. For this purpose, the study used the BASC (Behavior Assessment System for Children) set of tests, the DSM V hyperactivity checklist of and the Caras-R test. These tests were applied to a sample of 80 children who had a preterm birth (clinical sample), and 80 children were born at full term (control sample). The information collected was analyzed using the SPSS ${ }^{T M}$ software Version 22 and additional supporting software for the neuropsychological assessment of human behavior. The results showed that there were more cases of inattention or impulsivity in those born prematurely. This is manifested in their homes through atypical behavior, hyperactivity symptoms, episodes of isolation and the typical symptomatology of depression and somatization.

Key words: Prematurity, behavior, emotions, TDAH, attention (Social Sciences Unesco Thesaurus).

\section{Influência do nascimento prematuro nos processos comportamentais e emocionais de crianças de ensino básico.}

- Resumo (analítico): A pesquisa procurou determinar as principais características comportamentais que podem ocorrer em crianças na faixa etária de 6 a 10 anos de idade com nascimento prematuro. Foi utilizada a bateria de testes BASC (Do inglês Behavior Assessment System for Children), os critérios de verificação de hiperatividade do DSM-5 e o teste de Faces-R. A avaliação foi feita em 80 crianças prematuras (amostra clínica) e 80 crianças não prematuras (amostra controle). Os dados foram analisados utilizando o software estatístico SPSS versão 22 e o software de apoio para avaliação neuropsicológica da conduta humana. Em relação ao TDAH foram mais freqüentes os casos de desatenção ou impulsividade no grupo de crianças nascidas prematuramente, as quais são percebidas nas suas casas como crianças com comportamentos atípicos, com sintomas de hiperatividade, episódios de isolamento, sintomatologia relacionada com depressão e sintomas de somatização.

Palavras chave: prematuridade, conduta, emoções, TDAH, atenção (Thesaurus de Ciências Sociais da Unesco).

-1. Introducción. -2. Metodología. -3. Resultados. -4. Discusión. -5. Conclusiones. -6. Limitaciones. -7. Agradecimientos. -Lista de referencias.

\section{Introducción}

El nacimiento prematuro es tema de gran interés para la comunidad científica, especialmente por las características biológicas $\mathrm{y}$ funcionales de esta condición, y surgen interrogantes sobre las secuelas que puede dejar en un niño o niña el hecho de haber nacido prematuro. Según datos de la Organización Mundial de la Salud (OMS, 2013), estos tienen mayor tendencia a desarrollar discapacidades que serán manifestadas a lo largo de toda su vida (Kinney, Howson, McDougall, \& Lawn, 2012).
Diversos autores coinciden en afirmar que los partos prematuros son considerados una de las causas de la mortalidad infantil (Calderón, Vega, Velásquez, Morales, \& Vega, 2005; Faneite, 2000), representan el $75 \%$ de la mortalidad neonatal y casi la mitad de la morbilidad neurológica a largo plazo (Egan, Gutiérrez, Cuevas, \& Lucio, 2008). Como lo refieren Ríos-Flórez, Villegas-Vanegas y Marín-Rivera (2016), la niñez es una etapa relevante en el desarrollo de todo ser humano, la cual requiere de un correcto acompañamiento y conocimiento de todos los procesos que se presentan durante 
esta, incluyendo las condiciones que rodean el momento del nacimiento, particularmente en las gestaciones prematuras.

Pese a lo anterior, los índices de mortalidad disminuyen en la medida en que aumentan las semanas de nacimiento; así, un bebé que nace de 36 semanas tendría más posibilidades de sobrevivir que uno que nace a las 28 semanas (Hübner \& Ramírez, 2002). Según un estudio del Departamento Administrativo Nacional de Estadística (Dane, Colombia) el 11.4\% de la población nace prematura, cifra próxima a la conseguida por la OMS, que representa un poco más de 100000 partos prematuros al año, siendo el Eje Cafetero (Colombia) la zona con más altos índices, como lo menciona Palencia (2010); así mismo, define que el nacimiento prematuro es todo aquel que acontezca antes de la semana 37 y con menos de 2500 gramos de peso. Abordando los criterios de la OMS (2013), se ha clasificado a los niños y niñas pretérmino como prematuro general a quien nace antes de la semana 37 , prematuro tardío a quien nace entre la semana 34 con 0/7 días y la semana 36 con 6/7 días; quienes nacen antes de la semana 32 serían muy prematuros, mientras que serían extremadamente prematuros quienes nacen antes de la semana 28.

Por su parte, la Academia Americana de Pediatría propone como definición de prematurez: "aquellos recién nacidos menores de 38 semanas, debido al mayor riesgo de morbilidad existente entre las 37 y 38 semanas" (Salinas et al., 2006). Si bien cualquier alteración pre, peri o posnatal puede afectar el desarrollo neuropsicológico de niños y niñas, entre estos son comunes el retraso cognitivo, el déficit visoperceptivo, las dificultades en áreas motoras y los procesos de memoria, por lo que se afecta más adelante el aprendizaje y la capacidad de orientación espacial (FeliuIglesias, 2013); a nivel comportamental, existe mayor prevalencia al trastorno por déficit de atención con híperactividad (TDAH) y, si no es detectado tempranamente, puede aumentar su severidad, poniendo de manifiesto, tanto en niños como en niñas, conductas poco adaptativas a lo largo de su vida (Fernández, Fershteyn, Rains, \& De Ver Dye, 2003; Pallás, 2004; Poeta \& Rosa, 2006).
Es de considerar que la conducta en un individuo es producto de su actividad cerebral propia de los lóbulos frontales, que se encargan de la regulación, programación y ejecución de la conducta humana. Para el desarrollo adecuado del sistema nervioso es necesario tener en cuenta variables como la alimentación, la salud, el bienestar propio, la cultura, la educación, entre otras, las cuales son de gran importancia en este proceso de desarrollo, y pueden generar consecuencias en el funcionamiento cognitivo y conductual del niño o niña (Garófalo, Gómez, Vargas, \& Novoa, 2009; Mansilla, Acosta, \& Villazón, 2014; Rosselli, Matute, \& Ardila, 2010). En este sentido, Cavell (2000) y Hack (2005) consideran que la conducta es uno de los procesos que con mayor frecuencia puede verse afectado principalmente en la edad escolar; un asunto importante es que no siempre las secuelas del individuo nacido pretérmino se prevén durante los dos primeros años de vida.

Como concluye Hack (2005) en su investigación, los prematuros que no refieren alteraciones dentro de los dos primeros años de vida, pueden presentarlas entre los seis y siete años; por el contrario, las dificultades del neurodesarrollo manifestadas a los dos años, pueden no prolongarse en edades posteriores. En consecuencia, es relevante considerar la importancia de hacer en ambos periodos (infancia temprana y tardía) una valoración neuropsicológica, puesto que esta ayuda al diagnóstico e identificación del daño cerebral o de la disfunción neuropsicológica y, así mismo, recolectar datos sobre el nivel de gravedad del trastorno y las futuras consecuencias del paciente (Manga, \& Ramos, 2001; Rosselli, Matute, \& Ardila, 2010).

En las últimas décadas se le ha otorgado relevancia a temas relacionados con aspectos conductuales como lo es el TDAH, uno de los trastornos diagnosticados con mayor frecuencia y calificado como el síndrome infantil más controversial (Abdalá, 2014; American Psychiatric Association, 2014; Forns, Amador, \& Balanzó, 2001). Por otra parte, los niños y niñas prematuros manifiestan dificultades según el nivel de inmadurez de sus diferentes sistemas orgánicos $\mathrm{y}$, a su vez, dificultades para adaptarse al mundo externo, lo que va 
enlazado con el grado de prematurez; por esto, el cerebro inmaduro sería incapaz de integrar los estímulos que recibe al nacimiento, lo que llevaría a una desorganización cerebral que persistiría a lo largo de los años (Lackman, Capewell, Richardson, Da silva, \& Gagnon, 2001; Palencia, 2010; Sánchez-Rodríguez, Quintero-Villegas, Rodríguez-Camelo, NietoSanjuanero, \& Rodríguez-Balderrama, 2010; Yabar, 2006).

De forma similar, se ha planteado que entre los seis y los ocho meses de gestación hay mayor probabilidad de lesiones cerebrales, puesto que en dicho periodo se logra un incremento del 35\% del tamaño del cerebro; también aumenta cinco veces el volumen de la sustancia blanca, se efectúa la maduración estructural, se da un incremento en la conexión neuronal, se presenta la arborización dendrítica, entre otros procesos; así, el sistema nervioso del prematuro es más propenso a sufrir lesiones de orden neurológico debido a la interrupción de su desarrollo (Rodríguez, Vivas, Cangelosi, \& Schapira, 2008; Rosas, Velásquez, \& Criollo, 2014). En 2003, la revista Pediatrics publicó un artículo de Peterson et al. (2003), mencionando las áreas cerebrales comprometidas en niños prematuros; estos autores hallaron que las regiones parieto-occipitales mostraron menor cantidad de sustancia gris, al igual que en las sensorio-motoras y la corteza occipital inferior, pero aumentó en el cuerno central, cuerno occipital, y cuernos temporales de los ventrículos laterales; además, encontraron que la sustancia blanca, en las áreas parietooccipitales, tenía mayor volumen del lado izquierdo que del derecho.

El volumen de sustancia blanca en las regiones sensorio-motora y temporal media se interrelaciona significativamente con los resultados del desarrollo neurológico; así mismo, lesiones en el lóbulo frontal ocasionan dificultades en la formación de actitudes, en la autorregulación y en las conductas adaptativas, puesto que la corteza frontal se encarga del juicio y planeación para optimizar el resultado de la conducta (Clark, Boutros, \& Méndez, 2012; Peterson et al., 2003). Por su parte, RíosFlórez y Cardona-Agudelo (2016a) destacan la importancia de estudiar las regiones corticales en el procesamiento de las emociones, particularmente de áreas prefrontales, resaltando que el desarrollo emocional no se limita a procesos subcorticales referidos al sistema límbico.

Autores como Matas (2004), Pallas (2004) y Ruiz (2004), coinciden en afirmar que un parto prematuro no solo implica dificultades para los niños y niñas, sino que involucra en este proceso el bienestar de los padres, ya que ambos requieren del tiempo suficiente para prepararse física y psicológicamente: los primeros para lograr una adecuada maduración biológica, y los segundos para adoptar estrategias de afrontamiento frente a la llegada de un niño o niña prematuro (Irirarte \& Carrión, 2013).

Desde este punto, se debe comprender al niño o niña y sus dificultades, en conjunto con las áreas familiar, social, escolar y personal, con el propósito de conocer e intervenir los problemas que puedan presentar estos dentro de dichos espacios (Rosselli, Matute, \& Ardila 2010). Con frecuencia, las modificaciones en el ambiente familiar y escolar o el apoyo psicológico para niños y niñas conllevan a que el problema resulte lo menos limitante posible (De La Barra, Toledo, \& Rodríguez, 2002; Iriarte, \& Carrión, 2013); de igual forma, es importante considerar la prolongación del mismo cuidado que se ha conferido durante el embarazo al feto, hasta después de su nacimiento (Álvarez, 2011), para disminuir el posible impacto y potencializar las capacidades que debido a su desarrollo cerebral interrumpido han sido afectadas (York \& Devoe, 2002).

La literatura refiere diferentes estudios que han intentado correlacionar el neurodesarrollo y las implicaciones de carácter conductual en niños y niñas prematuros, encontrando que la edad de cinco y seis años es un referente al realizar los estudios, afirmándose en algunos de ellos que es en estas edades donde se inician los procesos de escolarización; es en esta etapa donde pueden originarse problemas de conducta, impulsividad y alteraciones autorregulatorias, lo cual puede incidir en el desarrollo $\mathrm{y}$ rendimiento académico, social y afectivo (Benítez, \& González, 2008; Rodríguez, Vivas, Cangelosi, \& Schapira, 2008; Turrini, Enumo, Ferrão, \& Monteiro, 2010). 
Un estudio de corte prospectivo, realizado en Francia en el 2008 con niños y niñas prematuros cuando tenían cinco años, determinó que los nacidos prematuros tenían problemas conductuales y emocionales, tendencia a desarrollar hiperactividad de tipo inatenta y bajo desempeño académico en comparación con niños y niñas nacidos a término; también influían otras variables como la salud mental de la madre y el estado de salud general al nacer (Delobel-Ayoub et al., 2009; Jain, 2008; Jasso-Gutiérrez, 2008; Raju, Higgins, Stark, \& Leveno, 2006).

Igualmente en Suiza, en 2015, se evaluaron niños y niñas de cinco años de edad con antecedente de prematuridad, en donde la cognición se estudió con el K-ABC y el comportamiento de las fortalezas y dificultades con el cuestionario SDQ. Los resultados arrojados se asociaron significativamente con las puntuaciones de hiperactividad del SDQ (coeficiente de 0.81, p <0.04), pero no con las puntuaciones cognitivas, los trastornos del neurodesarrollo o la necesidad de terapia (Graz, Tolsa, \& Fumeaux, 2015).

De forma similar, en 2012 fue publicado en la Revista Argentina de Ciencias del Comportamiento un estudio sobre las alteraciones neuropsicológicas y emocionales en niños y niñas prematuros de bajo peso (Bermúdez et al., 2012), concluyendo que los nacidos prematuros a la edad de siete y ocho años presentan alteraciones neuropsicológicas, principalmente relacionadas con memoria, función ejecutiva de conducta y atención. Igualmente, en Australia en 2009 se encontró que el principal problema conductual hallado fue de carácter atencional en prematuros extremos y con muy bajo peso al nacer (Spittle et al., 2009).

En relación con la conducta y la prematurez, un estudio liderado por investigadores de la Universidad de Warwick en Reino Unido, en más de 200 niños y niñas de seis años de edad que habían nacido antes de la semana 26 de gestación, se halló alteraciones de la conducta, problemas de tipo emocional, hiperactividad, atención e inadecuada relación con sus padres, afectando a los niños y niñas prematuros tanto en sus casas como en el colegio (Samara,
Marlow, \& Wolke, 2008). Por otra parte, Stephens y Vohr (2009) y Johnson et al. (2010), reportan que el peso al nacer influye sobre la inatención e hiperactividad de niños y niñas que han nacido con muy bajo y extremo bajo peso, manifestado por padres y profesores de niños y niñas en edad escolar (ocho a 12 años), y los índices fueron de $23 \%$ a $27 \%$ en niños y niñas nacidos con muy bajo peso, y del $33 \%$ al $37 \%$ en niños y niñas nacidos con extremo bajo peso.

En vista de las manifestaciones neuropsicológicas halladas en los niños y niñas nacidos pretérmino, estas se han comenzado a investigar desde temprana edad. Así lo demuestra un estudio sobre el efecto del nacimiento prematuro en el desarrollo cognitivo, psicomotor y socioafectivo realizado en España con niños y niñas de 18 meses de edad. Los resultados arrojaron que los nacidos prematuros tienen un mayor riesgo de presentar problemas cognitivos, atencionales y de regulación emocional, los que podrían generar dificultades en el periodo escolar (Monzóa et al., 2010).

En Colombia no ha sido relegada la variable del peso, adelantándose algunos estudios que exponen la incidencia de esta y la condición de prematurez en el neurodesarrollo. Muestra de ello es una investigación liderada por Gómez, Espinosa, Galvis, Chaskel y Ruiz (2015), realizada en el Hospital Militar de Bogotá, donde se pauta que los niños y niñas con nacimiento prematuro tienen un mayor riesgo de presentar alteraciones en el desarrollo y el comportamiento, siendo mayor en quienes al nacer mostraron un peso menor de 1500 gr y en los nacidos antes de la semana 32, correlacionando las dificultades en el neurodesarrollo con los problemas comportamentales.

Así mismo, en la Universidad Pontificia Javeriana de Bogotá (2005), se realizó un estudio acerca del desarrollo neurocomportamental del niño o niña recién nacido prematuro de 34 a 40 semanas, demostrando su capacidad para adaptarse y responder adecuadamente al medio ambiente. La diferencia más notable fue entre las semanas 35 y 39 de vida, siendo esta última en la que la mayoría de los bebés mostraron avances. Dichos resultados podrían 
tenerse en cuenta como predictores en la edad escolar, donde parece ser que se agudizan las manifestaciones conductuales y cognitivas (Garzón, Machuca, \& Riaño, 2005).

En este sentido, Pallás (2004) y Alonso, de la Cruz y López (2000) afirman que es necesario hacer seguimiento desde el mismo momento del nacimiento de un niño o niña de muy bajo peso y, si es posible, incluso antes. A pesar del énfasis investigativo centrado en los primeros años de vida, en la etapa adolescente se siguen manifestando las secuelas que deja la interrupción del desarrollo cerebral a causa del nacimiento pretérmino; en esta etapa se ha logrado evidenciar problemas de agresividad, comportamiento delincuencial o déficit de atención (Hille et al., 2001). Por su parte, RíosFlórez y Cardona-Agudelo (2016b) plantean que la condición de prematurez afecta el desarrollo integral de niños y niñas en ambientes académicos y de interacción social, teniendo estas alteraciones conductuales repercusión en el desempeño escolar.

De forma similar, en una investigación liderada por la Revista Mexicana de Neurociencias en 2005, en pacientes entre los tres y los 12 años diagnosticados con TDAH, se llegóa la conclusión de que factores preexistentes como asfixia perinatal, prematurez, secuelas de traumatismo craneoencefálico y procesos infecciosos, eran condicionantes al momento de diagnosticar el trastorno (Abdalá, 2014; Serrano, \& Hernández, 2005). Otros autores como Muñoz-Pérez, Pérez-García, Arroyo, Vichido-Luna y Soto-Vega (2014) en México, lideraron un estudio con el fin de contrastar los factores de riesgo perinatal en los niños y niñas con déficit de atención, con o sin hiperactividad (TDA/H), versus niños y niñas sanos, llegando a la conclusión de que el $22 \%$ de aquellos con TDAH nacieron prematuramente, mientras en el grupo control únicamente hubo un $8 \%$ de niños y niñas prematuros, mostrándose una diferencia estadística significativa entre ambos grupos.

En consideración a lo abordado hasta aquí, y al poco consenso y evidencia empírica de compromiso neuropsicológico en la prematurez, en esta investigación nos proponemos establecer las principales características conductuales y emocionales que pueden presentar niños y niñas entre seis a diez años de edad con antecedente de nacimiento prematuro, aspectos a valorarse en contextos familiares y educativos, a fin de promover la creación de programas y diseñar métodos de intervención que favorezcan el adecuado desarrollo de esta población y el entendimiento de sus características particulares al momento de la crianza y el abordaje escolar.

\section{Metodología}

La investigación está comprendida desde un enfoque cuantitativo, con diseño comparativocorrelacional; seleccionamos la muestra de clase por conglomerados bajo modelo no probabilístico o dirigido, tomando los criterios de Hernández, Fernández y Baptista (2014). Analizamos los datos obtenidos en la recolección de información producto del protocolo aplicado mediante estadística descriptiva-correlacional, empleando el software estadístico SPSS ${ }^{\mathrm{TM}}$ (IBM, versión 22, 2013). Ejecutamos la prueba de identificación de normalidad de los datos e igualdad de varianzas de Kolmogorov-Smirnov con la corrección de significación de Lilliefors; pruebas paramétricas a $\mathrm{T}$ de Student, no paramétricas a U de Mann-Whitney y Kruskal Wallis; coeficientes de correlación de Pearson y Spearman; Chí cuadrado; frecuencias y descriptivos generales.

Participantes: conformamos dos grupos, el primero compuesto por 80 niños y niñas de seis a diez años de edad, escolarizados, con antecedente clínico de nacimiento prematuro; y otro grupo de 80 niños y niñas en condición no clínica, de seis a diez años de edad, nacidos a término $\mathrm{y}$ escolarizados, equitativos en género. Los miembros del grupo control fueron seleccionados bajo las condiciones par de género, edad y nivel escolar de cada miembro de la muestra clínica; es decir, si un niño prematuro es de género masculino, con siete años de edad y segundo grado primaria, su par sería no prematuro y debía cumplir con estas mismas condiciones a fin de aumentar la confiabilidad de los datos analizados. La muestra total se encuentra entre los seis y los diez años de edad y entre primero y quinto grado escolar. Los niños y niñas del grupo clínico presentan como 
rango de semanas de gestación, entre la 26 y la 36. Los sujetos participantes no debían estar en condición alguna de discapacidad de carácter neuropsicológico, mental o físico (diferente a los posibles síntomas asociados a la condición de prematurez), ni estar recibiendo algún tipo de medicación o tratamiento preventivo, profiláctico o de prescripción continua. La muestra clínica inicial fue de 340 niños en condición de prematurez a partir de la cual, tras la construcción y revisión de la historia clínica, de los antecedentes y criterios de exclusión, se redujo a la muestra finalmente empleada.

Instrumentos: los test y pruebas empleados para llevar a cabo la investigación fueron la batería de pruebas BASC (sistema de evaluación de la conducta de niños y adolescentes) de los autores Reynolds y Kamphaus, versión 2004 (BASC-IBM, 2004), que evalúa aspectos conductuales y emocionales en los ámbitos escolares y familiares. La escala BASC analiza una amplia gama de dimensiones distintas (cada una de las presentadas en las tablas de resultados); además de evaluar trastornos emocionales, conductuales y problemas de personalidad, identifica rasgos positivos $y$ negativos que puedan orientar un tratamiento. La escala y sus componentes cuentan con alta consistencia interna y fiabilidad test-retest (95\%), así como diversos índices de validez por contenido. Empleamos para la calificación el software Basc Enhanced Assist ${ }^{\mathrm{TM}}$ versión 1.1, con baremos para Colombia estandarizados por el Grupo de Neuropsicología y Conducta de la Universidad de San Buenaventura, el Grupo de Neurociencias de la Universidad de Antioquia, el Instituto Neurológico de Antioquia (Indea) y la Universidad de Georgia - a partir de la primera versión de la prueba (Kamphaus et al., 2000) —. De igual forma, empleamos la prueba Caras-R de Thurstone (Yela \& Luque, 2012, edición 11), con fiabilidad interna del $95 \%$ y validez de contenido convergentedivergente, así como referida a un criterio externo del evaluando, el criterio TDAH que arroja y el índice de control de impulsividad, así como la lista de chequeo de los criterios de hiperactividad-impulsividad-inatención de la guía de consulta de los criterios diagnósticos del DSM-5 (APA, 2014).
Procedimiento: las evaluaciones las realizamos en tres sesiones de aproximadamente dos horas cada una; al inicio de cada una buscamos verificar el estado atencional y emocional de niños y niñas, y controlar las variables contextuales y ambientales que pudieran interferir en la aplicación de las pruebas Caras-R y el cuestionario BASC de autoevaluación. El cuestionario BASC (cuadernillo cuidadores en dos ambientes) y la lista de chequeo del DSM-5, los aplicamos por igual a padres, madres y docentes.

Consideraciones éticas: los procedimientos que adelantamos en la presente investigación los diseñamos de conformidad con las directrices estipuladas por el Ministerio de Salud Nacional de Colombia (Resolución No. 8430 de 1993) sobre la investigación con participantes humanos y el nivel de riesgo que implica; por lo tanto, el estudio se cataloga como de riesgo mínimo, debido a que no se puso en riesgo la integridad física o psicológica de los individuos participantes. Adicionalmente, la investigación se ajustó a las disposiciones estipuladas por el código ético y deontológico del psicólogo (Ley 1090 del 2006), con relación a la protección de la identidad, buen nombre, participación voluntaria y fines estrictamente académico-investigativos de los resultados de investigación. Así, la presente investigación la realizamos bajo la aprobación de los sujetos participantes y de su representante legal, a través del consentimiento informado individual, firmado de manera previa a la realización del procedimiento de aplicación. Para lo anterior, se les explicó en qué consistía el procedimiento y qué implicaciones tenía el hecho de aceptar participar en el mismo, el carácter voluntario de la participación y la posibilidad de abandono del estudio con previo aviso.

\section{Resultados}

Este apartado condensa un análisis objetivo y numérico desde parámetros estadísticos obtenidos, cuya funcionalidad y visión neuropsicológica - de lo que representa cualitativamente - abordamos en los apartados siguientes. 
Tabla 1. Datos biométricos.

\begin{tabular}{lcccccc}
\hline \multicolumn{1}{c}{ Criterio } & Grupo & M & DE & Q1 & Q3 & Sig. \\
\hline \multirow{2}{*}{ Edad } & 1 & 8.09 & 1.37 & 7.00 & 9.00 & \multirow{2}{*}{$0.964^{\mathrm{U}}$} \\
\multirow{2}{*}{ Semanas de gestación } & 2 & 8.09 & 1.31 & 7.00 & 9.00 & \\
& 1 & 34.06 & 2.68 & 33.00 & 36.00 & \multirow{2}{*}{$0.000^{* * * \mathrm{U}}$} \\
Escolaridad & 2 & 39.34 & 1.01 & 39.00 & 40.00 & \\
& 1 & 3.03 & 1.34 & 2.00 & 4.00 & \multirow{2}{*}{$0.827^{\mathrm{U}}$} \\
\hline
\end{tabular}

Nota. Grupo 1: muestra clínica; Grupo 2: muestra control; M: media; DE: desviación estándar; Q: cuartil; u: de MannWhitney; *** $: \leq 0.001$.

Los datos contenidos en la Tabla 1 en relación con variables sociodemográficas reflejan que, para el criterio de edad, la comparación de medias evidencia que los datos son equivalentes con variación mínima en la DE, lo que estadísticamente no refiere diferencias significativas siendo las dos muestras equivalentes. Para la variable semanas de gestación hay una diferencia de cinco semanas en relación con el promedio y una desviación estándar dentro del rango de normalidad de los prematuros, refiriendo estadísticamente relevancia la no equivalencia para este criterio, tal como lo esperábamos. Para la variable escolaridad hay una diferencia de dos niveles de escolaridad en cuanto a la comparación de medias, con variación no significativa en desviación estándar, lo que estadísticamente no muestra relevancia, ya que ambas son equivalentes. 
Tabla 2. Criterios conductuales y emocionales del autoinforme de la batería BASC (Sistema de evaluación de la conducta de niños y adolescentes).

\begin{tabular}{|c|c|c|c|c|c|c|}
\hline Criterio & Grupo & M & DE & $\mathbf{r}^{1}$ & $\mathbf{r}^{2}$ & Sig. \\
\hline \multirow{2}{*}{ Actitud hacia los profesores } & 1 & 5.39 & 14.83 & $-0.607 * * \mathrm{e}$ & $0.230^{* \mathrm{e}}$ & \multirow{2}{*}{$0.686^{\mathrm{U}}$} \\
\hline & 2 & 2.94 & 2.59 & $0.276^{* \mathrm{~s}}$ & $-0.076^{e}$ & \\
\hline \multirow{2}{*}{ Ansiedad } & 1 & 8.51 & 3.43 & $-0.197^{e}$ & $0.269 * \mathrm{~s}$ & \multirow{2}{*}{$0.060^{\mathrm{U}}$} \\
\hline & 2 & 7.16 & 3.69 & $0.089^{\mathrm{e}}$ & $-0.042^{e}$ & \\
\hline \multirow{2}{*}{ Depresión } & 1 & 8.13 & 12.39 & $-0.562 * * \mathrm{e}$ & $0.221 * \mathrm{e}$ & \multirow{2}{*}{$0.524^{\mathrm{U}}$} \\
\hline & 2 & 6.18 & 4.86 & $0.213^{\mathrm{s}}$ & $-0.044^{e}$ & \\
\hline \multirow{2}{*}{ Atipicidad } & 1 & 6.06 & 5.13 & $-0.253 * \mathrm{e}$ & $0.138^{\mathrm{s}}$ & \multirow{2}{*}{$0.201^{\mathrm{U}}$} \\
\hline & 2 & 5.21 & 4.76 & $0.251 * \mathrm{~s}$ & $-0.037^{e}$ & \\
\hline \multirow{2}{*}{ Locus de control } & 1 & 6.24 & 3.27 & $-0.244 * \mathrm{e}$ & $0.100^{\mathrm{e}}$ & \multirow{2}{*}{$0.064^{\mathrm{U}}$} \\
\hline & 2 & 5.26 & 3.23 & $-0.163^{\mathrm{s}}$ & $-0.029^{\mathrm{s}}$ & \\
\hline \multirow{2}{*}{ Estrés social } & 1 & 5.84 & 4.72 & $-0.477 * * \mathrm{e}$ & $0.212^{\mathrm{e}}$ & \multirow{2}{*}{$0.090^{\mathrm{U}}$} \\
\hline & 2 & 4.93 & 3.95 & $0.130^{\mathrm{s}}$ & $-0.077^{\mathrm{e}}$ & \\
\hline \multirow{2}{*}{ Sensación de inadecuación } & 1 & 5.20 & 5.50 & $-0.539 * * \mathrm{e}$ & $0.204^{\mathrm{e}}$ & \multirow{2}{*}{$0.066^{\mathrm{U}}$} \\
\hline & 2 & 3.64 & 2.52 & $0.100^{\mathrm{s}}$ & $-0.025^{\mathrm{e}}$ & \\
\hline \multirow{2}{*}{ Actitud hacia el colegio } & 1 & 6.09 & 13.31 & $-0.622 * * \mathrm{e}$ & $0.198^{e}$ & \multirow{2}{*}{$0.062^{\mathrm{U}}$} \\
\hline & 2 & 3.41 & 3.67 & $0.277 * \mathrm{~s}$ & $-0.256 * \mathrm{~s}$ & \\
\hline \multirow{2}{*}{ Relación con padres } & 1 & 8.30 & 2.20 & $-0.130^{\mathrm{e}}$ & $-0.069^{e}$ & \multirow{2}{*}{$0.975^{\mathrm{U}}$} \\
\hline & 2 & 8.25 & 2.38 & $-0.108^{e}$ & $0.070^{\mathrm{s}}$ & \\
\hline \multirow{2}{*}{ Autoconfianza } & 1 & 12.65 & 6.11 & $-0.023^{\mathrm{s}}$ & $0.070^{\mathrm{e}}$ & \multirow{2}{*}{$0.563^{\mathrm{U}}$} \\
\hline & 2 & 12.66 & 5.68 & $0.162^{\mathrm{s}}$ & $0.084^{\mathrm{s}}$ & \\
\hline \multirow{2}{*}{ Autoestima } & 1 & 11.04 & 10.92 & $-0.050^{\mathrm{s}}$ & $0.037^{\mathrm{e}}$ & \multirow{2}{*}{$0.485^{\mathrm{U}}$} \\
\hline & 2 & 10.41 & 9.92 & $0.198^{\mathrm{e}}$ & $-0.060^{\mathrm{e}}$ & \\
\hline \multirow{2}{*}{ Relaciones interpersonales } & 1 & 9.29 & 4.35 & $0.048^{\mathrm{e}}$ & $-0.050^{\mathrm{e}}$ & \multirow{2}{*}{$0.168^{\mathrm{C}}$} \\
\hline & 2 & 9.31 & 3.31 & $0.126^{\mathrm{e}}$ & $-0.044^{e}$ & \\
\hline
\end{tabular}

Nota. Grupo 1: muestra clínica; Grupo 2: muestra control; M: media; DE: desviación estándar; Q: cuartil; " : de MannWhitney; ${ }^{*}: \mathrm{p} \leq 0.05 ;{ }^{* *}: \mathrm{p} \leq 0.01 ; \mathrm{r}^{1}:$ correlación en relación a semanas de gestación; $\mathrm{r}^{2}$ : correlación en relación a edad; ${ }^{\mathrm{e}}$ : coeficiente de correlación de Pearson; ${ }^{\text {s: }}$ coeficiente de correlación de Spearman.

Los datos reflejados anteriormente muestran que la variable actitud hacia los profesores, en relación con el promedio, presenta correlación para las semanas de gestación y edad respecto a la variable; sin embargo, entre grupos reportan correlación positiva. En cuanto a la variable ansiedad, esta presenta un comportamiento inverso entre los grupos clínico y no clínico para la variable semanas de gestación y edad: a menor número de semanas de gestación para el grupo clínico y a menor edad para el grupo no clínico se presenta mayor sintomatología característica de ansiedad, siendo inversos estos dos mismos criterios intragrupo sin que se halle diferencia estadística entre grupos. La percepción de los niños y niñas hacia sí mismos, para la mayoría de las variables entre grupos, no presenta diferencias desde el punto de vista estadístico; 
sin embargo, las correlaciones intragrupo de los diferentes criterios con el criterio de edad y semanas de gestación reportan resultados variables que requieren de análisis individual detallado.

Tabla 3. Criterios de valoración por profesores.

\begin{tabular}{|c|c|c|c|c|c|c|}
\hline Criterio & Grupo & M & DE & r 1 & r 2 & Sig. \\
\hline \multirow{2}{*}{ Ansiedad } & 1 & 5.54 & 4.57 & $0.280 * \mathrm{e}$ & $-0.081^{\mathrm{s}}$ & \multirow{2}{*}{$0.099^{\mathrm{U}}$} \\
\hline & 2 & 5.61 & 4.81 & $-0.079^{e}$ & $0.228 * \mathrm{e}$ & \\
\hline \multirow{2}{*}{ Problemas atencionales } & 1 & 8.74 & 4.48 & $0.242 * \mathrm{e}$ & $-0.163^{\mathrm{s}}$ & \multirow{2}{*}{$0.444^{\mathrm{U}}$} \\
\hline & 2 & 7.80 & 4.14 & $0.242^{\mathrm{s}}$ & $0.143^{\mathrm{e}}$ & \\
\hline \multirow{2}{*}{ Agresión } & 1 & 9.48 & 8.40 & $0.203^{\mathrm{e}}$ & $0.158^{\mathrm{e}}$ & \multirow{2}{*}{$0.872^{\mathrm{U}}$} \\
\hline & 2 & 9.49 & 7.93 & $0.128^{\mathrm{s}}$ & $0.070^{\mathrm{e}}$ & \\
\hline \multirow{2}{*}{ Atipicidad } & 1 & 7.16 & 8.10 & $0.187^{\mathrm{e}}$ & $-0.045^{\mathrm{s}}$ & \multirow{2}{*}{$0.661^{\mathrm{U}}$} \\
\hline & 2 & 6.88 & 7.86 & $0.016^{\mathrm{e}}$ & $0.273 * \mathrm{~s}$ & \\
\hline \multirow{2}{*}{ Aislamiento } & 1 & 5.41 & 3.76 & $0.231 * \mathrm{e}$ & $-0.133^{\mathrm{s}}$ & \multirow{2}{*}{$0.949^{\mathrm{U}}$} \\
\hline & 2 & 5.60 & 4.07 & $0.135^{\mathrm{s}}$ & $0.160^{\mathrm{e}}$ & \\
\hline \multirow{2}{*}{ Depresión } & 1 & 6.24 & 5.56 & $0.192^{\mathrm{s}}$ & $-0.044^{\mathrm{s}}$ & \multirow{2}{*}{$0.945^{\mathrm{U}}$} \\
\hline & 2 & 6.46 & 5.69 & $0.104^{\mathrm{e}}$ & $0.077^{\mathrm{e}}$ & \\
\hline \multirow{2}{*}{ Problemas conducta } & 1 & 3.75 & 4.41 & $0.207^{\mathrm{e}}$ & $0.031^{\mathrm{e}}$ & \multirow{2}{*}{$0.796^{\mathrm{U}}$} \\
\hline & 2 & 4.15 & 4.80 & $0.018^{\mathrm{e}}$ & $0.132^{\mathrm{e}}$ & \\
\hline \multirow{2}{*}{ Problemas aprendizaje } & 1 & 9.00 & 4.63 & $0.252 * \mathrm{~s}$ & $-0.180^{\mathrm{s}}$ & \multirow{2}{*}{$0.029 * \mathrm{U}$} \\
\hline & 2 & 7.40 & 4.80 & $-0.020^{\mathrm{e}}$ & $0.109^{\mathrm{e}}$ & \\
\hline \multirow{2}{*}{ Somatización } & 1 & 4.99 & 4.81 & $0.139^{\mathrm{s}}$ & $0.088^{\mathrm{e}}$ & \multirow{2}{*}{$0.320^{\mathrm{U}}$} \\
\hline & 2 & 4.56 & 4.89 & $-0.063^{\mathrm{s}}$ & $0.068^{\mathrm{e}}$ & \\
\hline \multirow{2}{*}{ Hiperactividad } & 1 & 12.85 & 7.95 & $0.167^{\mathrm{e}}$ & $0.094^{\mathrm{s}}$ & \multirow{2}{*}{$0.307^{\mathrm{U}}$} \\
\hline & 2 & 11.28 & 6.86 & $0.023^{\mathrm{s}}$ & $0.187^{\mathrm{e}}$ & \\
\hline \multirow{2}{*}{ Habilidades en el estudio } & 1 & 15.81 & 5.55 & $0.085^{\mathrm{s}}$ & $0.201^{\mathrm{s}}$ & \multirow{2}{*}{$0.004 * u$} \\
\hline & 2 & 18.35 & 5.29 & $-0.004^{\mathrm{S}}$ & $0.112^{\mathrm{e}}$ & \\
\hline \multirow{2}{*}{ Compañerismo } & 1 & 12.69 & 3.73 & $-0.037^{\mathrm{s}}$ & $0.203^{\mathrm{e}}$ & \multirow{2}{*}{$0.002 * * U$} \\
\hline & 2 & 14.63 & 4.40 & $-0.063^{\mathrm{s}}$ & $0.220 * \mathrm{e}$ & \\
\hline \multirow{2}{*}{ Habilidades sociales } & 1 & 16.73 & 5.28 & $0.166^{\mathrm{e}}$ & $0.007^{\mathrm{e}}$ & \multirow{2}{*}{$0.000 * * * c$} \\
\hline & 2 & 19.78 & 5.58 & $-0.124^{\mathrm{S}}$ & $0.205^{\mathrm{s}}$ & \\
\hline \multirow{2}{*}{ Adaptabilidad } & 1 & 10.86 & 2.66 & $-0.241 * \mathrm{~s}$ & $-0.038^{e}$ & \multirow{2}{*}{$0.546^{\mathrm{U}}$} \\
\hline & 2 & 11.11 & 2.54 & $-0.029^{\mathrm{s}}$ & $-0.102^{e}$ & \\
\hline
\end{tabular}

Nota. Grupo 1: muestra clínica; Grupo 2: muestra control; M: media; DE: desviación estándar; Q: cuartil; u: de MannWhitney, ${ }^{*}: \mathrm{p} \leq 0.05 ;{ }^{* *}: \mathrm{p} \leq 0.01 ; * *: \mathrm{p} \leq 0.001 ; \mathrm{r}^{1}$ : correlación en relación a semanas de gestación; $\mathrm{r}^{2}$ : correlación en relación a edad; e: coeficiente de correlación de Pearson; s: coeficiente de correlación de Spearman. 
Los análisis estadísticos para la tabla 3 en relación con los criterios habilidades en el estudio, compañerismo y habilidades sociales presentan diferencias significativas entre grupos a nivel de $\mathrm{p} \leq 0.01$, y el criterio problemas de aprendizaje con un $95 \%$ de diferencias entre grupo; los demás criterios referidos a la percepción del profesor hacia el estudiante no presentan diferencias entre grupos, al comparar la equivalencia de las medias. A nivel de las correlaciones para las variables de edad y semanas de gestación, en relación con los criterios medidos, se presentan relaciones dispares que ameritan análisis individuales.

Tabla 4. Criterios de valoración por padres.

\begin{tabular}{|c|c|c|c|c|c|c|}
\hline Criterio & Grupo & $\mathbf{M}$ & DE & r 1 & $\mathbf{r} 2$ & Sig. \\
\hline \multirow{2}{*}{ Atipicidad } & 1 & 6.36 & 4.20 & $0.041^{\mathrm{s}}$ & $-0.063^{\mathrm{S}}$ & \multirow{2}{*}{$0,054^{* U}$} \\
\hline & 2 & 5.15 & 3.89 & $0.239 * \mathrm{e}$ & $0.020^{\mathrm{s}}$ & \\
\hline \multirow{2}{*}{ Problemas atención } & 1 & 8.89 & 3.23 & $0.024^{\mathrm{e}}$ & $0.030^{\mathrm{s}}$ & \multirow{2}{*}{$0.087^{\mathrm{U}}$} \\
\hline & 2 & 7.86 & 3.38 & $0.015^{\mathrm{s}}$ & $-0.061^{\mathrm{s}}$ & \\
\hline \multirow{2}{*}{ Hiperactividad } & 1 & 11.99 & 4.78 & $0.140^{\mathrm{e}}$ & $-0.127^{e}$ & \multirow{2}{*}{$0.046 * \mathrm{U}$} \\
\hline & 2 & 10.64 & 5.55 & $0.068^{\mathrm{s}}$ & $-0.079^{e}$ & \\
\hline \multirow{2}{*}{ Ansiedad } & 1 & 11.06 & 4.71 & $-0.046^{\mathrm{e}}$ & $0.105^{\mathrm{e}}$ & \multirow{2}{*}{$0.070^{\mathrm{t}}$} \\
\hline & 2 & 9.75 & 4.36 & $0.0163^{\mathrm{s}}$ & $-0.047^{e}$ & \\
\hline \multirow{2}{*}{ Aislamiento } & 1 & 6.06 & 3.48 & $-0.145^{\mathrm{s}}$ & $-0.049^{e}$ & \multirow{2}{*}{$0.026^{* U}$} \\
\hline & 2 & 4.89 & 3.05 & $0.140^{\mathrm{s}}$ & $0.018^{\mathrm{e}}$ & \\
\hline \multirow{2}{*}{ Depresión } & 1 & 7.89 & 5.61 & $0.139^{\mathrm{s}}$ & $0.070^{\mathrm{e}}$ & \multirow{2}{*}{$0.050 * \mathrm{U}$} \\
\hline & 2 & 6.55 & 5.13 & $0.072^{\mathrm{s}}$ & $-0.056^{e}$ & \\
\hline \multirow{2}{*}{ Problemas de conducta } & 1 & 5.21 & 3.83 & $-0.065^{\mathrm{s}}$ & $0.071^{\mathrm{s}}$ & \multirow{2}{*}{$0.386^{\mathrm{U}}$} \\
\hline & 2 & 4.84 & 3.69 & $0.175^{\mathrm{s}}$ & $0.073^{\mathrm{s}}$ & \\
\hline \multirow{2}{*}{ Somatización } & 1 & 7.60 & 5.39 & $-0.173^{e}$ & $0.311 * * \mathrm{~s}$ & \multirow{2}{*}{$0.018 * * \mathrm{U}$} \\
\hline & 2 & 5.76 & 4.64 & $0.197^{\mathrm{s}}$ & $0.062^{\mathrm{s}}$ & \\
\hline \multirow{2}{*}{ Agresión } & 1 & 9.25 & 5.60 & $0.158^{\mathrm{e}}$ & $-0.027^{\mathrm{s}}$ & \multirow{2}{*}{$0.388^{\mathrm{U}}$} \\
\hline & 2 & 7.91 & 4.74 & $0.028^{\mathrm{s}}$ & $0.113^{\mathrm{s}}$ & \\
\hline \multirow{2}{*}{ Compañerismo } & 1 & 18.65 & 6.01 & $-0.101^{e}$ & $-0.007^{e}$ & \multirow{2}{*}{$0.076^{\mathrm{U}}$} \\
\hline & 2 & 20.16 & 5.67 & $-0.177^{\mathrm{s}}$ & $-0.117^{e}$ & \\
\hline \multirow{2}{*}{ Habilidades sociales } & 1 & 24.41 & 8.28 & $-0.119^{\mathrm{s}}$ & $0.051^{\mathrm{s}}$ & \multirow{2}{*}{$0.281^{\mathrm{U}}$} \\
\hline & 2 & 25.79 & 7.56 & $0.062^{\mathrm{s}}$ & $-0.038^{\mathrm{s}}$ & \\
\hline \multirow{2}{*}{ Adaptabilidad } & 1 & 12.93 & 3.43 & $-0.020^{\mathrm{s}}$ & $-0.128^{e}$ & \multirow{2}{*}{$0.257^{\mathrm{U}}$} \\
\hline & 2 & 13.53 & 3.70 & $0.016^{\mathrm{e}}$ & $-0.042^{\mathrm{s}}$ & \\
\hline
\end{tabular}

Nota. Grupo 1: muestra clínica; Grupo 2: muestra control; M: media; DE: desviación estándar; Q: cuartil; ": de Mann-Whitney; t: T de Student; *: $\mathrm{p} \leq 0.05 ;{ }^{* *}: \mathrm{p} \leq 0.01 ; \mathrm{r}^{1}$ : correlación en relación a semanas de gestación; $\mathrm{r}^{2}$ : correlación en relación a edad; ${ }^{\text {e: }}$ coeficiente de correlación de Pearson; s: coeficiente de correlación de Spearman.

Los resultados de la tabla 4 reflejan que el criterio de atipicidad presenta diferencias estadísticamente significativas, según la perspectiva de los padres del grupo clínico en relación con la visión de los padres del grupo no clínico hacia sus hijas e hijos. Para este 
mismo criterio - aun cuando no significativoencontramos una relación inversamente proporcional para el grupo clínico en el que a menor edad mayor atipicidad. Los criterios de hiperactividad (aislamiento, depresión y somatización) presentan diferencias significativas entre grupos, según la concepción de los padres; es de resaltar que para el dato hiperactividad, la variable edad para ambos grupos presenta relaciones inversamente proporcionales. Los demás datos clínicos contemplados por la escala son concordantes desde la perspectiva de los padres entre grupos; es decir, la puntuación media sugiere que no hay diferencias significativas entre ellos.

Tabla 5. Concordancia de criterios conductuales y emocionales entre padres y profesores.

\begin{tabular}{lcccccc}
\hline \multicolumn{1}{c}{ Criterio } & Grupo & $\mathbf{M}$ & $\mathbf{D E}$ & $\mathbf{r}^{\mathbf{1}}$ & $\mathbf{r}^{2}$ & Sig. \\
\hline Padres & 1 & 7.50 & 5.38 & $0.190^{\mathrm{e}}$ & $0.197^{\mathrm{s}}$ & $0.181^{\mathrm{U}}$ \\
Inatención & 2 & 6.48 & 5.11 & $0.063^{\mathrm{s}}$ & $0.065^{\mathrm{s}}$ & \\
Hiperactividad & 1 & 8.51 & 6.63 & $0.106^{\mathrm{e}}$ & $0.023^{\mathrm{s}}$ & $0.122^{\mathrm{U}}$ \\
& 2 & 6.68 & 5.29 & $-0.045^{\mathrm{e}}$ & $0.099^{\mathrm{s}}$ & \\
Combinado & 1 & 9.73 & 10.90 & $0.097^{\mathrm{e}}$ & $0.062^{\mathrm{s}}$ & $0.055^{* \mathrm{U}}$ \\
& 2 & 6.28 & 7.26 & $-0.087^{\mathrm{s}}$ & $0.121^{\mathrm{s}}$ & \\
Profesores & 1 & 7.78 & 5.46 & $0.100^{\mathrm{e}}$ & $-0.023^{\mathrm{s}}$ & $0.005^{* * \mathrm{U}}$ \\
Inatención & 2 & 5.26 & 4.56 & $0.090^{\mathrm{e}}$ & $0.106^{\mathrm{e}}$ & \\
& 1 & 8.28 & 5.89 & $0.117^{\mathrm{e}}$ & $0.050^{\mathrm{s}}$ & $0.005^{* * \mathrm{U}}$ \\
Hiperactividad & 2 & 5.63 & 4.73 & $0.071^{\mathrm{s}}$ & $0.101^{\mathrm{s}}$ & \\
& 1 & 8.81 & 11.31 & $-0.087^{\mathrm{s}}$ & $-0.030^{\mathrm{e}}$ & $0.018^{* * \mathrm{U}}$ \\
Combinado & 2 & 4.80 & 7.67 & $-0.016^{\mathrm{s}}$ & $0.071^{\mathrm{s}}$ & \\
\hline
\end{tabular}

Nota. Grupo 1: muestra clínica; Grupo 2: muestra control; M: media; DE: desviación estándar; Q: cuartil; $\mathrm{r}^{1}$ : correlación en relación a semanas de gestación; $\mathrm{r}^{2}$ : correlación en relación a edad; ${ }^{\text {e }}$ coeficiente de correlación de Pearson; $*$ : $\mathrm{p} \leq 0.05$; $* *: \mathrm{p} \leq 0.01 ;$; : coeficiente de correlación de Spearman.

Los análisis de la tabla 5 evidencian diferencias significativas entre grupos para la variable mixta, en relación con los criterios diagnósticos referentes a inatenciónhiperactividad-impulsividad, entre los grupos clínico y no clínico, presentándose con mayor frecuencia en los niños y niñas prematuros. Por otra parte, los criterios de inatención, hiperactividad-impulsividad, reflejan presencia de los síntomas en igual medida estadística para ambos grupos, según la perspectiva de los padres y madres. De otro lado, estos mismos tres criterios presentan diferencias estadísticamente significativas a nivel de $\mathrm{p}<0.01$ desde la visión de los profesores entre ambos grupos para cada criterio. 
Tabla 6. Correlación entre padres y profesores para los criterios del BASC y del Checklist TDAH DSM-5.

\begin{tabular}{|c|c|c|}
\hline Criterio & Grupo & r Checklist \\
\hline \multirow{2}{*}{ Inatención } & 1 & $0.418^{\mathrm{e}}$ \\
\hline & 2 & $0.253 * \mathrm{e}$ \\
\hline \multirow{2}{*}{ Hiperactividad } & 1 & $0.593^{\mathrm{s}}$ \\
\hline & 2 & $0.375 * * \mathrm{e}$ \\
\hline \multirow{3}{*}{ Combinado } & 1 & $0.491^{\mathrm{e}}$ \\
\hline & 2 & $0.378 * * \mathrm{e}$ \\
\hline & & $\mathrm{r}$ Basc \\
\hline \multirow{2}{*}{ Atipicidad } & 1 & $0.271^{\mathrm{s}}$ \\
\hline & 2 & $0.315 * * \mathrm{~s}$ \\
\hline \multirow{2}{*}{ Problemas atención } & 1 & $0.145^{\mathrm{s}}$ \\
\hline & 2 & $0.321 * * \mathrm{e}$ \\
\hline \multirow{2}{*}{ Hiperactividad } & 1 & $0.143^{\mathrm{e}}$ \\
\hline & 2 & $0.186^{\mathrm{s}}$ \\
\hline \multirow{2}{*}{ Ansiedad } & 1 & $0.144^{\mathrm{e}}$ \\
\hline & 2 & $0.082^{\mathrm{s}}$ \\
\hline \multirow{2}{*}{ Aislamiento } & 1 & $-0.017^{e}$ \\
\hline & 2 & $0.175^{\mathrm{s}}$ \\
\hline \multirow{2}{*}{ Depresión } & 1 & $0.428^{\mathrm{s}}$ \\
\hline & 2 & $0.180^{\mathrm{e}}$ \\
\hline \multirow{2}{*}{$\begin{array}{l}\text { Problemas de } \\
\text { conducta }\end{array}$} & 1 & $0.313^{\mathrm{s}}$ \\
\hline & 2 & $0.377 * * \mathrm{~s}$ \\
\hline \multirow{2}{*}{ Somatización } & 1 & $0.328^{\mathrm{e}}$ \\
\hline & 2 & $0.249 * \mathrm{~s}$ \\
\hline \multirow{2}{*}{ Agresión } & 1 & $0.241^{\mathrm{s}}$ \\
\hline & 2 & $0.360 * * \mathrm{~s}$ \\
\hline \multirow{2}{*}{ Compañerismo } & 1 & $0.142^{\mathrm{s}}$ \\
\hline & 2 & $0.149^{\mathrm{e}}$ \\
\hline \multirow{2}{*}{ Habilidades sociales } & 1 & $0.162^{\mathrm{s}}$ \\
\hline & 2 & $0.322 * * \mathrm{~s}$ \\
\hline \multirow{2}{*}{ Adaptabilidad } & 1 & $0.108^{\mathrm{e}}$ \\
\hline & 2 & $0.054^{\mathrm{s}}$ \\
\hline
\end{tabular}

Nota. Grupo 1: muestra clínica; Grupo 2: muestra control; ${ }^{\text {e: }}$ coeficiente de correlación de Pearson; ${ }^{\text {s: }}$ coeficiente de correlación de Spearman; r BASC: correlación entre padres y profesores para los criterios de la escala BASC; r Checklist: correlación entre padres y profesores para los criterios de TDAH del DSM-5; $: \mathrm{p} \leq 0.05 ; * *: \mathrm{p} \leq 0.01$.

Para el grupo clínico, es decir, el compuesto por niños y niñas prematuros, los análisis evidenciados en la tabla 6 reflejan concordancia entre la visión de los padres y los profesores hacia los niños; por tanto, no hay diferencias significativas desde el punto de vista estadístico para todos los criterios. No obstante, para el grupo de niños y niñas nacidos a término, 
los criterios de inatención, hiperactividad, inatención-hiperactividad-impulsividad, atipicidad, problemas de atención, problemas de conducta, somatización, agresión y habilidades sociales, presentan diferencias significativas entre la concepción de padres y profesores hacia estos niños. La tabla 6 también permite establecer correlaciones entre los criterios de atención y conducta de la escala BASC y los de inatención e hiperactividad de la Checklist de DSM-5, entre lo manifestado por padres, madres y docentes.

Tabla 7. Subtipos de TDAH, frecuencias y relación entre grupos.

\begin{tabular}{lccc}
\hline \multicolumn{1}{c}{ Criterio } & G. Clínico & G. No clínico & Total N \\
\hline Inatento & 8 & 6 & 14 \\
Impulsivo & 27 & 19 & 46 \\
Combinado & 6 & 9 & 15 \\
Sin TDAH & 39 & 46 & 85 \\
Sig. & \multicolumn{2}{c}{$0.415^{\mathrm{K}}$} & 160 \\
\hline
\end{tabular}

Nota. G. Clínico: grupo 1; G. No clínico: grupo 2; ${ }^{\text {k }}$ prueba no paramétrica de Kruskal Wallis para coeficiente de significancia $(\mathrm{p}), *: \mathrm{p} \leq 0.05, * *: \mathrm{p} \leq 0.01$.

Los descriptivos de la tabla 7 según los resultados de la prueba de atención Caras-r, en relación con el criterio de TDAH arrojado por la misma, visualizan mayor frecuencia de casos de inatención o impulsividad por separado en los niños prematuros, y mayor frecuencia de ambos síntomas en los niños de nacimiento a término; sin embargo, para los resultados obtenidos en esta prueba no son significativas estas diferencias a nivel de frecuencias de casos en cada criterio.

\section{Discusión}

A partir de los objetivos propuestos para la investigación, los resultados ponen de manifiesto diferencias significativas entre los niños y niñas nacidos a término y los niños y niñas de nacimiento pretérmino, en las funciones evaluadas $y$ descritas de forma particular y relacionados con cuadros conductuales, emocionales, procesos atencionales, problemas de aprendizaje, habilidades sociales y de adaptación, así como de reconocimiento de actitudes y aptitudes percibidas por los propios niños y niñas, por sus padres, madres o sujetos cuidadores y docentes.

Desde este punto, en relación con los componentes conductuales, esta investigación encontró presencia de criterios de déficit de atención con hiperactividad de tipo combinado en los niños y niñas con nacimiento pretérmino, en comparación con los no prematuros, lo que concuerda con lo planteado por Raju et al. (2006) y Delobel-Ayoub et al. (2009), quienes hallaron que los niños y niñas prematuros tenían problemas conductuales, predominancia de hiperactividad e inatención. De igual forma, Samara, Marlow y Wolke (2008), hallaron en quienes nacieron prematuros un exceso considerable de alteraciones en la conducta, incluyendo problemas de hiperactividad y atención, afectándolos tanto en sus casas como en el colegio. Sin embargo, en este estudio se encuentra que los criterios de solo inatención o solo hiperactividad se reportan en el ámbito académico y no en el hogar, contrario a lo planteado por estos autores.

En entornos escolares y familiares, como lo enunciamos en el párrafo anterior, se identifica presencia de síntomas mixtos del TDAH en los niños y niñas prematuros más que en los nacidos a término, y no únicamente hiperactividad o solo inatención en cada entorno. En relación con los criterios únicos de inatención, los resultados de esta investigación concuerdan con Bermúdez et al. (2012): principalmente alteraciones en la atención, para las actividades desarrolladas en el ámbito escolar. Aunado a lo anterior, en el estudio desarrollado en México 
por Muñoz-Pérez et al. (2014), se plantea que, contrastando la condición de prematurez con alteraciones conductuales, el 22\% de los niños y niñas con TDAH nacieron prematuramente, mientras en el grupo control hubo un $8 \%$ de niños y niñas prematuros, mostrándose una diferencia estadística significativa entre ambos grupos. Los datos de la presente investigación concuerdan con estos autores al referir mayor presencia de criterios diagnósticos para TDAH en población de nacimiento pretérmino.

De otro lado, los resultados hallados en esta investigación plantean mayores problemas y dificultades de aprendizaje en los niños y niñas de nacimiento pretérmino, asociados de igual forma a la cantidad de semanas de gestación; para este aspecto, dichos resultados se asemejan a los encontrados por Raju et al. (2006), Delobel-Ayoub et al. (2009) y Monzóa et al. (2010), llegando a la conclusión de que los niños y niñas pretérmino presentan un bajo desempeño académico y alteraciones en el aprendizaje; por otra parte, los datos del presente estudio sugieren que los niños y niñas prematuros presentan menores habilidades en el estudio, lo cual se relaciona con la presencia de dichos problemas de aprendizaje, también referidos por Ríos-Flórez y Cardona-Agudelo (2016b).

Porotraparte, retomando los planteamientos de Raju et al. (2006), Delobel-Ayoub et al. (2009) y Monzóa et al. (2010), en relación con la regulación emocional, estos autores plantean problemas de tipo emocional en los niños y niñas prematuros; no obstante, no establecen particularidades o detalles en relación con el tipo de inconvenientes. La investigación descrita en este artículo nos permitió encontrar concordancia con la presencia de problemas emocionales en niños y niñas de nacimiento prematuro, y nos posibilita ser específicos identificando principalmente mayores conductas de aislamiento, sintomatología característica de depresión y cuadros de somatización en esta población de interés; por otra parte, no hay datos significativos indicadores de sintomatología de ansiedad o problemas conductuales relacionados con la agresión, diferentes a los asociados usualmente a la infancia.
Así mismo, y en relación con los componentes emocionales, en esta investigación encontramos que, para el ámbito socioafectivo, los niños y niñas de nacimiento pretérmino presentan mayores dificultades en el desarrollo de habilidades sociales y de compañerismo identificadas en ambientes sociales y familiares. A su vez, no presentan dificultad en la adaptabilidad a entornos sociales o académicos, en comparación con los niños y niñas de nacimiento a término, aun cuando su desempeño sea más individual que de interacción con pares o adultos. En relación con planteamientos como este, hay que decir que no se encuentran actualmente datos en la literatura que posibiliten discutir sobre ello, por lo que se pueden considerar los primeros aportes al tema, por lo menos en cuanto a su especificidad y puntualidad del criterio valorado. En esta investigación logramos obtener datos y análisis que conllevan a ser abordarlos a modo de conclusión de resultados.

\section{Conclusiones}

Esta investigación supone aportes considerables al campo de la neuropsicología y la psicología en general, y a disciplinas de las neurociencias interesadas en conocimientos relacionados con la infancia desde el punto de vista cognitivo, conductual y de funcionamiento cerebral, particularmente producto de este estudio, en relación con consecuencias de interferencias $\mathrm{o}$ alteraciones a nivel pre $\mathrm{y}$ perinatal.

Así, la cantidad de semanas de gestación de los niños y niñas pretérmino pareciera no ser un factor influyente en la magnitud de las alteraciones conductuales, emocionales o de aprendizaje; es decir, sin importar, por ejemplo, si la gestación fue de 29 o 33 semanas, la presencia o no de alteraciones es por igual a nivel de la condición de prematurez al compararse con sus pares no prematuros. Así mismo, al tener en cuenta la variable edad cronológica en los niños y las niñas de nacimiento pretérmino, la edad actual de estos sí es un factor influyente en el desarrollo de una u otra alteración o dificultad a nivel de conducta y estados emocionales desde un punto de vista general. 
De igual forma, los sujetos de la muestra clínica (es decir, de nacimiento prematuro) no presentan autodeterminación y autoreporte de dificultades emocionales, conductuales o de aprendizaje en relación con sus pares no clínicos, desde el nivel de conciencia de dichas dificultades. Desde un punto de vista detallado de los resultados obtenidos, encontramos que al considerar el número de semanas de gestación, los niños y las niñas prematuros extremos presentan una mayor actitud negativa hacia sus docentes. Concluimos igualmente que, en relación con la autopercepción, a mayor edad cronológica en la época de escolaridad primaria, los niños y niñas prematuros presentan mayor sintomatología característica de ansiedad y depresión; sin embargo, en relación con esta última, los niños y niñas prematuros extremos son los que presentan mayor sintomatología y mayores comportamientos atípicos.

Los niños y niñas nacidos muy prematuros se perciben a sí mismos con menor autocontrol, mayor estrés social, sensación de inadecuación $\mathrm{y}$ una mejor actitud hacia el colegio, en relación con los prematuros con más semanas de nacimiento. Por otra parte, en el entorno escolar se encuentra que a mayor semanas de gestación dentro de la prematurez, los niños son percibidos con mayores conductas de sintomatología de ansiedad, problemas de aprendizaje y atencionales, aislamiento social y una menor adaptabilidad a este entorno; por su parte, los niños y niñas pretérmino no presentan conductas de agresividad, somatización o dificultades en las habilidades en el estudio en pro de las dificultades presentadas; tampoco es detectado por los profesores y profesoras sintomatología alguna relacionada con depresión. En un entorno familiar de nacimiento prematuro, estos son percibidos con mayores conductas atípicas, síntomas de hiperactividad, episodios de aislamiento y sintomatología característica de depresión y cuadros de somatización. En estos últimos encontramos que, a mayor edad cronológica, estos niños y niñas presentan mayores síntomas; también son percibidos como poseedores de habilidades de adaptación y socialización a este entorno.

En este mismo sentido, y al realizar la revisión de los criterios para el diagnóstico de TDAH, hallamos que los padres, madres y docentes concuerdan con la presencia de síntomas de este trastorno de tipo combinado; para los tipos de TDAH inatento o de hiperactividad-impulsividad, son los profesores quienes reportan más casos aislados en estos; por tanto, en el cumplimiento de los criterios clínicos para TDAH, el $7.5 \%$ de los niños prematuros presentan TDAH tipo combinado, $10 \%$ de tipo inatento y $33.75 \%$ de tipo hiperactividadimpulsividad. Encontramos entonces que la condición de nacimiento prematuro es un factor influyente y considerable en el desarrollo de conductas, emociones y actitudes particulares que impactan de forma negativa en la mayoría de los criterios valorados y que, a su vez, en otros no es un factor determinante de conductas y habilidades que se desarrollan de forma similar a sus pares no prematuros.

Es así que para concebir la relación cerebroconducta en su totalidad, el niño debe estar enmarcado dentro de su entorno sociocultural que involucra su desarrollo y determina las diferentes maneras de intervención en materia de daño cerebral, particularmente el que se puede suscitar ante la condición de nacimiento pretérmino, induciendo a alteraciones en la correcta maduración y funcionamiento de los lóbulos frontales y las conexiones que de estos se generen hacia el resto del sistema nervioso, principalmente con el sistema límbico. En este punto y desde el referente teórico, aun cuando los registros por neuroimagen o los antecedentes clínicos no evidencian alteraciones estructurales en el cerebro de niños de nacimiento pretérmino, áreas involucradas en el desarrollo de los procesos neuropsicológicos pueden verse comprometidas en funcionalidad y conectividad. Por tanto, al abordar temáticas relacionadas con Neuropsicología, esta debe ser considerada como una disciplina científica de las neurociencias que aborda el estudio del pensamiento, la cognición, las emociones y la conducta humana, como producto de la actividad cerebral, y que toma como base de estudio la funcionalidad normal o alteración del sistema nervioso, su organización anatómica y fisiológica y las interacciones de este con todo el organismo. 


\section{Limitaciones}

El poco material científico y reciente limita establecer un panorama discutible sobre las variables y temáticas aquí analizadas y la población abordada con contexto actual, por lo cual son escasos los estudios recientes que contemplen una metodología similar a la aquí empleada o a los constructos considerados; de igual forma, la mayoría de las investigaciones publicadas se limitan a plantear resultados y conclusiones generales en cuanto a la conducta y las emociones, reduciéndose ampliamente al TDAH, sin contemplar estados conductuales y emocionales de otraíndole.Por ellolos resultados específicos hallados en esta investigación los exponemos como conclusión al no tener referente para discutir. De otro lado, aún cuando las muestras empleadas eran homólogas en criterios demográficos y cronológicos, se encuentran reducidas en cantidad de sujetos, por lo que a futuras investigaciones el empleo de una muestra más amplia aumentaría la confiabilidad en la generalización de los datos, más allá de la población de referencia.

\section{Agradecimientos}

A las instituciones educativas, padres y profesores de los niños y niñas que conformaron la muestra de este estudio, y a estos mismos por su colaboración; y a los auxiliares de la investigación del grupo de estudio e investigación Hippocampus de la ciudad de Medellín.

\section{Lista de referencias}

Abdalá, A. L. (2014). Trastorno por déficit de atención e hiperactividad (TDAH): factores gestacionales y perinatales asociados. Evidencia Médica e Investigación en Salud, 22, 178-181. doi:10.1016/j. rmclc.2015.02.005

Alonso, C., De la Cruz, J., \& López, M. (2000). Apoyo al desarrollo de los niños nacidos demasiado pequeños, demasiado pronto. Revista de Neurología, 5(1), 30-39.

Álvarez, M. (2011). El apego como principal factor protector de la primera infancia.
Margen. Revista de Trabajo Social y Ciencias Sociales, (61), 9-5. Recuperado de: https://dialnet.unirioja.es/servlet/ articulo? codigo $=3756309$

American Psychiatric Association (2014). Guía de consulta de los criterios diagnósticos del DSM-5 ${ }^{\circledR}$ : Spanish Edition of the Desk Reference to the Diagnostic Criteria From $D S M-5^{\circledR}$. Washington, Londres: American Psychiatric Publishing.

BASC-IBM (2004). BASC: Sistema de evaluación de conductas para niños $y$ adolescentes. Versión 1.1. (BASC Enhanced plus, Assist $\left.{ }^{T M}\right)$ for IBM PC and compatible computers with a minimum of $640 \mathrm{~K}$ memory. Recuperado de: http:// www.pearsonclinical.com/support/ technical-support/assist-software/basc-2assist-software.html

Benítez, Y. R., \& González, M. N. (2008). Estudio neuropsicológico en niños de 6 años con antecedente de hipoxia perinatal. Arch Neurocien (Mex), 13(3), 162-169. Recuperado de: http://new. medigraphic.com/cgi-bin/resumen. cgi?IDARTICULO $=25828$

Bermúdez,O. G., Cruz-Quintana, F., Sosa, M. Á., Mañas, M., De la Cruz, J., \& Pérez-García, M. (2012). Alteraciones neuropsicológicas y emocionales en niños prematuros de muy bajo peso al nacer. Revista Argentina de Ciencias del Comportamiento, 4(2), 3-10. Recuperado de: www.psyche.unc.edu.ar/ racc

Calderón, J., Vega, G., Velásquez, J., Morales, R., \& Vega, A. (2005). Factores de riesgo materno asociados al parto pretérmino. Rev Med IMSS, 43(4), 339-342. Recuperado de: http://www.medigraphic.com/pdfs/ imss/im-2005/im054i.pdf

Cavell, T. A. (2000). Working with parents of aggressive children: A practitioner's guide. Washington: American Psychological Association (APA).

Clark, D. L., Boutros, N. N., \& Mendez, M. F. (2012). El cerebro y la conducta: neuroanatomía para psicólogos. México, D. F.: El Manual Moderno.

De La Barra, F., Toledo, V., \& Rodríguez, J. (2002). Estudio de salud mental en dos 
cohortes de niños escolares de Santiago occidente. I: prevalencia y seguimiento de problemas conductuales y cognitivos. Revista Chilena de Neuro-psiquiatría, 40(1), 9-21. Recuperado de: http://dx.doi. org/10.4067/S0717-92272002000400007.

Delobel-Ayoub, M., Arnaud, C., WhiteKoning, M., Casper, C., Pierrat, V., Garel, M., Larroque, B., Burguet, A. ... Larroque, B. (2009). Behavioral problems and cognitive performance at 5 years of age after very preterm birth: the Epipage Study. Pediatrics, 123(6), 1485-1492. Recuperado de: http://dx.doi.org/10.1542/ peds.2008-1216

Egan, L.A. V., Gutiérrez, A. K. C., Cuevas, M.P., \& Lucio, J. R. (2008). Perfil epidemiológico del parto prematuro. Ginecol Obstet Mex, 76(9), 542-548. Recuperado de: http:// www.medigraphic.com/pdfs/ginobsmex/ gom-2008/gom089h.pdf

Faneite, P. (2000). Análisis quinquenal de 30 años la mortalidad perinatal. Rev Obstet Ginecol Venez, 60, 23-25. Recuperado de: http://cania.msinfo.info/bases/biblo/texto/ pdf $2 /$ faneite.pdf

Feliu-Iglesias, N. (2013). La identificación de niños prematuros en educación infantil. (Tesis de maestría no publicada). Universidad Internacional de la Rioja, Logroño, España. Recuperado de: http:// reunir.unir.net/handle/123456789/1712

Fernández, I. D., Fershteyn, Z., Rains, A., \& De Ver Dye, T. (2003). Estudios recientes de valoración epidemiológica de la actividad física, el crecimiento fetal y el parto pretérmino: una revisión narrativa. Clínicas Obstétricas y Ginecológicas, (2), 395-402. Recuperado de: https://dialnet.unirioja.es/ servlet/articulo? codigo $=903624$

Forns, M., Amador, J. A., \& Balanzó, B. M. (2001). Trastorno por déficit de atención con hiperactividad: características del trastorno por déficit de atención con hiperactividad. Anuario de Psicologia/The UB Journal of Psychology, 32(4), 5-22. Recuperado de: http://revistes.ub.edu/index.php/Anuariopsicologia/article/viewFile/8791/11024

Garófalo, N., Gómez, A., Vargas, J., \& Novoa, L. (2009). Repercusión de la nutrición en el neurodesarrollo y la salud neuropsiquiátrica de niños y adolescentes. Revista Cubana de Pediatría, 81(2), 10-20. Recuperado de: http://scielo.sld.cu/scielo. php? script $=$ sci_arttextypid=S003475312009000200008 ylng=es.

Garzón, D., Machuca, A., \& Riaño, F. (2005). Desarrollo neurocomportamental del recién nacido prematuro de 34 a 40 semanas, asistente al programa madre canguro. (Trabajo de grado). Pontificia Universidad Javeriana, Bogotá, Colombia. Recuperado de: http:/hdl.handle. net/10554/7949

Gómez, J., Espinosa, E., Galvis, C., Chaskel, R., \& Ruiz, L. (2015). Caracterización clínica de los aspectos comportamentales relacionados con el neurodesarrollo en niños de 2 a 5 años con antecedente de prematurez en el Hospital Militar Central. Bogotá, D. C.: Universidad Militar Nueva Granada. Recuperado de: http://hdl.handle. net $/ 10654 / 12404$

Graz, M. B., Tolsa, J. F., \& Fumeaux, C. J. (2015). Being small for gestational age: Does it matter for the neurodevelopment of premature infants? A cohort study. PloS one, 10(5), e0125769. doi:10.1371/journal. pone. 0125769

Hernández, R., Fernández, C., \& Baptista, L. (2014). Metodología de la investigación. México, D. F.: McGraw-Hill Interamericana.

Hack, M. (2005). Escaso valor predictivo de las escalas de Bayley de desarrollo infantil para la función cognitiva en la edad escolar de niños recién nacidos de muy bajo peso. Pediatrics, 60(2), 81-89. Recuperado de: http://www.elsevier.es/ es-revista-pediatrics-10-articulo-escasovalor-predictivo-las-escalas-13114702

Hille, E. T., Den Ouden, A. L., Saigal, S., Wolke, D., Lambert, M., Whitaker, A. ... Paneth, N. (2001). Behavioural problems in children who weigh $1000 \mathrm{~g}$ or less at birth in four countries. The Lancet, 357(69), 1641-1643. Recuperado de: http://www. sciencedirect.com/science/article/pii/ S0140673600048182 
Hübner, M.E., \& Ramírez, R. (2002). Sobrevida, viabilidad y pronóstico del prematuro. Revista Médica de Chile, 130(8), 931-938. Recuperado de: http://dx.doi.org/10.4067/ S0034-98872002000800015

Iriarte, A., \& Carrión, M. (2013). Experiencias de los padres de grandes prematuros en la Unidad de Cuidado Intensivo Neonatal: revisión sistemática de la evidencia cualitativa. Metas de Enfermería, 16(2), 20-25. Recuperado de: http://www. enfermeria21.com/revistas/metas/ articulo/80416/

Jain, L. (2008). School outcome in late preterm infants: A cause for concern. The Journal of Pediatrics, 153 (1), 5-6. Recuperado de: $\quad$ http://dx.doi.org/10.1016/j. jpeds.2008.03.001

Jasso-Gutiérrez, L. (2008). ¿Habrá en el futuro un mayor número acumulado de individuos con secuelas neurológicas por haber nacido prematuros? Boletín Médico del Hospital Infantil de México, 65(5), 327-330. Recuperado de: http://www. scielo.org. $\mathrm{mx} / \mathrm{scielo} . \mathrm{php}$ ?pid=S1665$11462008000500001 \mathrm{yscript}=\mathrm{sci}$ arttextytlng=en

Johnson, S., Hollis, C., Kochhar, P., Hennessy, E., Wolke, D., \& Marlow, N. (2010). Psychiatric disorders in extremely preterm children: Longitudinal finding at age 11 years in the EPICure study. Journal of the American Academy of Child y Adolescent Psychiatry, 49(5), 453-463. Recuperado de: http://www.sciencedirect.com/science/ article/pii/S0890856710001425

Kamphaus, R. W., Jiménez, M. E., Pineda, D., Rowe, E. W., Fleckenstein, L., Restrepo, M. A. .. Palacio, L. G. (2000). Análisis transcultural de un instrumento de dimensiones múltiples en el diagnóstico del déficit de atención. Revista de Neuropsicología, Neuropsiquiatría y Neurociencias, 2(2), 51-63. Recuperado de: http://neurociencias.udea.edu.co/es/ research/cognitive/publications/show/171

Kinney, M. V., Howson, C. P., McDougall, L., \& Lawn, J. E. (2012). Resumen ejecutivo de Nacidos Demasiado Pronto: Informe de Acción Global sobre Nacimientos
Prematuros. Ginebra: March of Dimes, PMNCH, Save the Children, Organización Mundial de la Salud. Recuperado de: http://www.who.int/pmnch/media/ news/2012/borntoosoon_execsum_es.pdf

Lackman, F., Capewell, V., Richardson, B., Da silva, O., \& Gagnon, R. (2001). The risks of spontaneous preterm delivery and perinatal mortality in relation to size at birth according to fetal versus neonatal growth standards. American Journal of Obstetrics and Gynecology, 184(5), 946-953. Recuperado de: http://dx.doi. org/10.1067/mob.2001.111719

Manga, D., \& Ramos, F. (2001). Evaluación de los síndromes neuropsicológicos infantiles. Revista de Neurología, 32(7), 664-675. Recuperado de: http://www.neurologia. $\mathrm{com} / \mathrm{sec} /$ resumen.php? $\mathrm{id}=2000176$

Mansilla, R., Acosta, K., \& Villazon, A. (2014). Prematurez: nociones relevantes y riesgo neurológico del prematuro. Revista Desafios, 1(8), 51-60. Recuperado de: http://revistas.ut.edu.co/index.php/ desafios/article/view/461

Matas, A. J. (2004). III Jornada de la SPAPex. Oncología, 15, 2-38. Recuperado de: http://www.spapex.org/sites/default/files/ bol15.pdf

Monzóa, M. A., Giménez, A. O., Simó-Teufelb, S., Banaclocheb, D., Graciac, S. B., \& Celmac, M. A. (2010). P-5: el efecto del nacimiento pretérmino en el desarrollo cognitivo, psicomotor y socioafectivo. Revista Pediatría de Atención Primaria, 12 (supl19). Recuperado de:

http://scielo.isciii.es/scielo.php?pid=S113 976322010000500042yscript=sci_arttet

Muñoz-Pérez, M. J., Pérez-García, J. C., Arroyo, C., Vichido-Luna, M. Á., \& Soto-Vega, E. (2014). Factores de riesgo asociados con el déficit de atención en los niños con o sin hiperactividad. Revista Mexicana de Pediatría, 81(3), 89-92. Recuperado de: http://www.medigraphic.com/pdfs/pediat/ sp-2014/sp143b.pdf

Organización Mundial de la Salud-OMS (2013). Nota descriptiva $N^{\circ} 363$, alusiva a «Nacimientos Prematuros». Ginebra: Centro de prensa de la Organización 
Mundial de la Salud. Recuperado de: http:// www.who.int/mediacentre/factsheets/ fs363/es/

Palencia, A. (2010). "Parto prematuro". Revista Precop, 9(4), 10-19. Recuperado de: https://scp.com.co/precop/

Pallás, C. R. (2004). III Jornadas de actualización en Pediatría de atención Primaria de la SPAPex. Seguimiento neurológico del niño prematuro. Neonatología, Foro Pediátrico. 15(1), 4-10. Recuperado de: http://www. spapex.org/sites/default/files/bol15.pdf

Peterson, B. S., Anderson, A. W., Ehrenkranz, R., Staib, L. H., Tageldin, M., Colson, E., .. Ment, L. R. (2003). Regional brain volumes and their later neurodevelopmental correlates in term and preterm infants. Pediatrics, 111(5), 939-948. Recuperado de: http://pediatrics.aappublications.org/ content/111/5/939.short

Poeta, L., \& Rosa, F. (2006). Características biopsicosociales de los escolares con indicadores de trastorno de déficit de atención e hiperactividad. Revista de Neurología, 43(10), 584-588. Recuperado de: http://www.motricidade.com.br/pdfs/ edm/2006.6.pdf

Raju,T.N.,Higgins, R.D., Stark,A.R., \& Leveno, K. J. (2006). Optimizing care and outcome for late-preterm (near-term) infants: A summary of the workshop sponsored by the National Institute of Child Health and Human Development. Pediatrics, 118(3), 1207-1214. doi:10.1542/peds.2006-0018

Reynolds, C. R., \& Kamphaus, R. W. (2004). $B A S C$. Sistema de evaluación de conductas para niños y adolescentes: manual. Barcelona: TEA Ediciones. Recuperado de: www.teaediciones.com

Ríos-Flórez, J. A., \& Cardona-Agudelo, V. (2016a). Ruptura del paradigma subcortical en los procesos emocionales. Revista Poiésis, (31), 132-140. Recuperado de: http://www.funlam.edu.co/revistas/index. $\mathrm{php} /$ poiesis/article/view/2100/1607

Ríos-Flórez, J. A., \& Cardona-Agudelo, V. (2016b). Procesos de aprendizaje en niños de 6 a 10 años de edad con antecedente de nacimiento prematuro. Revista Latinoamericana de Ciencias Sociales,
Niñez y Juventud, 14(2), 1071-1085. doi:1 $0.11600 / 1692715 x .14213241115$

Ríos-Flórez, J. A., Villegas-Vanegas, S., \& Marín-Rivera,J.A.(2016). Neuropsicología de las praxias y procesos perceptuales en el niño de nacimiento pretérmino. Revista Argentina de Neuropsicología, 28(1), 1-21. Recuperado de: http://www.revneuropsi. sonepsa.com.ar/images/stories/pdf/ riosetalranps28.pdf

Rodríguez, G., Vivas, S., Cangelosi, M., \& Schapira, I. (2008). Avatares en prematuros de 3 a 5 años y sus madres. Revista del Hospital Materno Infantil Ramón Sardá, 27(4), 152-154. Recuperado de: http://www. redalyc.org/articulo.oa?id=91211281003

Rosas, S. M., Velásquez, K. S., \& Criollo, Á. R. (2014). Prematurez: nociones relevantes y riesgo neurológico del prematuro. Revistas Desafios, 1(8), 51-60. Recuperado de: Revistas.ut.edu.co/index.php/desafios/ article/download/461/388

Rosselli, M., Matute, E., \& Ardila, A. (2010). Neuropsicología del desarrollo infantil. México, D. F.: El Manual Moderno.

Salinas, H., Albornoz, J., Erazo, M., Catalán, J., Hübner, M. E., Preisler, J., \& Fernández, M. (2006). Impacto económico de la prematurez y las malformaciones congénitas sobre el costo de la atención neonatal. Revista Chilena de Obstetricia y Ginecología, 71(4), 234-238. Recuperado de: $\quad$ http://dx.doi.org/10.4067/S071775262006000400003

Samara, M., Marlow, N., \& Wolke, D. (2008). Pervasive behavior problems at 6 years of age in a total-population sample of children born at $\leq 25$ weeks of gestation. Pediatrics, 122(3), 562-573. Recuperado de: http:// dx.doi.org/10.1542/peds.2007-3231

Sánchez-Rodríguez, G., Quintero-Villegas, L. J., Rodríguez-Camelo, G., Nieto-Sanjuanero, A., \& Rodríguez-Balderrama, I. (2010). Disminución del estrés del prematuro para promover su neurodesarrollo: nuevo enfoque terapéutico. Medicina Universitaria, 12(48), 176-180. Recuperado de: http://eprints.uanl.mx/id/ eprint/8319 
Serrano, M., \& Hernández, G. (2005). Déficit de atención e hiperactividad. Revista Mexicana de Neurociencias, 6(2), 124128. Recuperado de: revmexneuroci.com

Spittle, A. J., Treyvaud, K., Doyle, L. W., Roberts, G., Lee, K. J., Inder, T. E., \& Anderson, P. J. (2009). Early emergence of behavior and social-emotional problems in very preterm infants. Journal of the American Academy of Child y Adolescent Psychiatry, 48(9), 909-918. doi: 10.1097/ CHI.0b013e3181af8235

SPSS-IBM (2013). SPSS para Windows. Versión 22. Illinois: Syntax Reference Guide. Recuperado de: http://www.spss. com

Stephens, B. E., \& Vohr, B. R. (2009). Neurodevelopmental outcome of the premature infant. Pediatric Clinics of North America, 56(3), 631-646. Recuperado de: http://dx.doi.org/10.1016/j. pcl.2009.03.005

Thurstone, L. L., Yela, M., \& Luque, T. (2012). Caras- $R$ : test de percepción de diferenciasrevisado: manual. Barcelona: Tea.

Turrini, F. A., Enumo, S. R., Ferrão, E. D., \& Monteiro, R. N. (2010). Comportamentos afetivo-motivacionais durante prova assistida diferenciam pré-escolares nascidos prematuros e com baixo peso dos nascidos a termo. Psicología: teoría e prática, 12(2), 158-172. Recuperado de: http://www.redalyc.org/articulo. oa? id=193817420011

Yabar, M. (2006). Seguimiento de 5 años de niños prematuros y de bajo peso nacidos en el año 2001 referidos de pediatría al Servicio de Medicina de RehabilitaciónNiños. Hospital Nacional Daniel A. Carrión-Callao. (Tesis de Especialización en Medicina no publicada). Universidad Nacional Mayor de San Marcos, Lima, Perú. Recuperado de: http://cybertesis. unmsm.edu.pe/handle/cybertesis/2453

York, J., \& Devoe, M. (2002). Health issues in survivors of prematurity. (Featured CMC Topic: Pediatrics). Southern Medical Journal, 95(9), 969-977. Recuperado de: http://www.gale.cengage.com 